Recepción: 20 / 01 / 2019

Aceptación: 19 / 03 / 2019

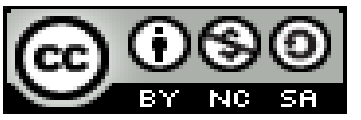

Ciencias de la salud

Publicación: 05 / 05 / 2019

Artículo de Revisión

\title{
Consideraciones frente la gravedad de problemas de esguince en el paciente
}

\section{Considerations against the gravity of sprain problems in the patient}

\section{Considerações contra a gravidade de problemas de aspiração no paciente}

Albert J. Macías-Peralta ${ }^{\mathrm{I}}$ josh_08@hotmail.com

Stephane P. Guerrero-Zambrano ${ }^{\text {II }}$ paologuerrerozambrano@gmail.com

Lisbeth E. Alvear-Toala ${ }^{\text {III }}$ estealve_15_2005@hotmail.com

Raisa S. Castro-Castro IV dra.rcastro33@gmail.com

Castulo L. Moreira-Moreira ${ }^{\mathrm{V}}$ castuloleonel@hotmail.com

José F. Macias-Riera ${ }^{\mathrm{VI}}$ freddyjr01@hotmail.com

\section{Correspondencia: josh_08@hotmail.com}

Médico Cirujano; Médico en Funciones Hospitalarias del Hospital Dr. Verdi Cevallos Balda; Portoviejo, Ecuador.

Médico Cirujano; Médico residente de cirugía del Hospital Verdi Cevallos Balda; Portoviejo, Ecuador. Médico Cirujano; Médico residente de cirugía del Hospital Verdi Cevallos Balda; Portoviejo, Ecuador. Médico Cirujano; Médico residente de cirugía del Hospital Verdi Cevallos Balda; Portoviejo, Ecuador. Médico Cirujano; Médico residente de cirugía del Hospital Verdi Cevallos Balda; Portoviejo, Ecuador. Médico Cirujano; Médico residente de cirugía del Hospital Verdi Cevallos Balda; Portoviejo, Ecuador. 


\title{
Resumen
}

El presente trabajo da lugar a una investigación realizada a través de una revisión bibliográfica de lo que es un esguince y de todo lo que este tipo de lesión conlleva. Un esguince va a depender de su origen, esto dará lugar a la edad y las condiciones del paciente influirán en gran medida; no será igual la consideración a tomar si es un paciente joven deportista a un adulto de mediana edad sedentario. El trabajo hace mención de forma detalla que esguinces son lesiones (rasgadura, torsión, distensión o estiramiento) que se presentan en los ligamentos de las articulaciones del cuerpo ya sea muñecas, rodillas, tobillos, dedos y en ocasiones puede presentarse un esguince de cuello (esguince cervical). El más común es el esguince de tobillo; aunque también le siguen de muñeca y de pulgar. El primero se presenta en grados: 1-leve, 2-moderado, 3-grave. Partiendo de esto variara la intensidad de la lesión y las consideraciones a tomar. Se conoce que el tratamiento más idóneo y efectivo para los esguinces es el reposo, hielo, compresión y elevación o sus siglas en ingles RICE. A pesar del tratamiento que le recete el medico al paciente; va a depender del paciente su recuperación. Esto puede influir en complicaciones a futuro o secuelas.

Palabras claves: Esguince; Lesión; Ligamento; Articulación; Tobillo.

\begin{abstract}
The present work gives rise to an investigation carried out through a bibliographic review of what is a sprain and everything that this type of injury entails. A sprain will depend on its origin, this will lead to age and the conditions of the patient will greatly influence; the consideration to be taken will not be equal if it is a young athlete patient to a sedentary middle-aged adult. The work makes mention in detail that sprains are injuries (ripping, twisting, stretching or stretching) that occur in the ligaments of the joints of the body, be it wrists, knees, ankles, fingers, and sometimes a neck sprain may occur (cervical sprain). The most common is the ankle sprain; although they also follow him wrist and thumb. The first is presented in degrees: 1-mild, 2-moderate, 3-severe. Based on this, the intensity of the injury and the considerations to be taken will vary. It is known that the most suitable and effective treatment for sprains is rest, ice, compression and elevation or its initials in English
\end{abstract}


Albert J. Macías-Peralta; Stephane P. Guerrero-Zambrano; Lisbeth E. Alvear-Tóala; Raisa S. Castro-Castro; Castulo L. Moreira-Moreira; José F. Macias-Riera

RICE. In spite of the treatment that the doctor prescribes to the patient; it will depend on the patient's recovery. This may influence future complications or sequelae.

Keys words: Sprain; Injury; Ligament; Articulation; Ankle.

\section{Resumo.}

O presente trabalho dá origem a uma investigação realizada por meio de uma revisão bibliográfica sobre o que é entorse e tudo o que esse tipo de lesão acarreta. Uma entorse dependerá da sua origem, isto levará à idade e as condições do paciente influenciarão muito; a consideração a ser tomada não será igual se for um paciente jovem atleta a um adulto sedentário de meia-idade. O trabalho menciona detalhes do formulário que entorses são lesões (rasgamento, a tensão de torção ou de alongamento) que ocorrem juntas do corpo do ligamento, quer pulsos, joelhos, os tornozelos, pés e, por vezes, pode apresentar um pescoço torcido (coluna cervical). O mais comum é a entorse do tornozelo; embora eles também o sigam pulso e polegar. O primeiro é apresentado em graus: 1-leve, 2-moderado, 3-grave. Com base nisso, a intensidade da lesão e as considerações a serem tomadas irão variar. Sabese que o tratamento mais adequado e eficaz para as entorses é o repouso, gelo, compressão e elevação ou suas iniciais no ARROZ inglês. Apesar do tratamento que o médico prescreve ao paciente; isso dependerá da recuperação do paciente. Isso pode influenciar futuras complicações ou sequelas.

Palavras chaves: Entorse Lesão Ligamento; Articulação; Tornozelo.

\section{Introducción.}

La palabra esguince viene del latín de la palabra exquintiare, la cual significa literalmente desgarrar (Martínez, 2019). Se puede definir un esguince como la rasgadura, torsión, distensión o estiramiento de un ligamento (tejido que une uno o más huesos de una articulación) lo que vendría siendo una lesión traumática frecuente en el aparato locomotor ocasionada por accidentes laborales, caídas, actividades recreativas o deportivas.

En el manual de primeros auxilios para enfermería se menciona que los esguinces más frecuentes ocurren en personas jóvenes y adultas. La localización más común de los 
esguinces es el tobillo y la rodilla, aunque pueden producirse en cualquier articulación con movimiento. (González García, 2013, pág. 128)

Como hace referencia (Méndez Flores, 2018) a que las articulaciones más vulnerables de sufrir esguinces son los tobillos, rodillas, dedos de la mano y muñeca.

Un esguince puede ocurrir cuando una persona se:

- Cae en un brazo

- Cae en un lado del pie

- Tuerce una rodilla.

Los esguinces más frecuentes son los de:

- Tobillo

- Rodilla

- Hombro

- Codo

Las causas de un esguince pueden variar desde una fuerte caída por falsear una pisada al momento de caminar hasta un movimiento brusco en la realización de una determinada actividad ya sea laboral, deportiva o recreativa. Este tipo de lesión puede ser de baja a moderada intensidad. Y es así como menciona el autor (Méndez Flores, 2018) cuando se refiere a que habitualmente en un traumatismo que origina un esguince o una contusión, el paciente puede referir que inicialmente no presentó ninguna limitación, apareciendo ésta al paso del tiempo siendo frecuente la expresión: "en caliente no me dolía". Y en ocasiones resulta difícil establecer una relación entre el momento del traumatismo y la aparición de los síntomas por el tiempo transcurrido, motivando ello cierta incertidumbre al paciente.

Los síntomas tienden a ser comunes en la mayoría de los esguinces: molestia y dolor en el área afectada, hematomas, hinchazón, incapacidad de usar el miembro afectado y en algunos casos deformación. 
Se debe tener en cuenta que los esguinces se clasifican de acuerdo al grado de la lesión; en la siguiente tabla se puede apreciar de forma detallada los grados de esguinces:

Tabla 1. Clasificación de los esguinces según su gravedad

\begin{tabular}{|c|c|}
\hline $\begin{array}{l}\text { Grado 1: leve } \\
\text { Lesión parcial de un ligamento en } \\
\text { la que las fibras del ligamento } \\
\text { están distendidas pero intactas. } \\
\text { La lesión es microscópica }\end{array}$ & $\begin{array}{l}\text { - Sin pérdida funcional o con limitación } \\
\text { leve } \\
\text { - El lesionado es capaz de utilizar la } \\
\text { articulación con apoyo total } \\
\text { - Dolor mínimo y sensibilidad leve al tacto } \\
\text { - Edema e inflamación leve } \\
\text { - No existe inestabilidad mecánica }\end{array}$ \\
\hline $\begin{array}{l}\text { Grado 2: moderado } \\
\text { Lesión incompleta de un } \\
\text { ligamento en la que algunas fibras } \\
\text { del ligamento están parcialmente } \\
\text { desgarradas. La lesión es parcial }\end{array}$ & $\begin{array}{l}\text { - Limitación parcial de la función y el } \\
\text { movimiento } \\
\text { - El lesionado tiene dolor cuando utiliza la } \\
\text { articulación } \\
\text { - Dolor y edema moderados. } \\
\text { - Equimosis de leve a moderada } \\
\text { - Edema sobre las estructuras afectadas } \\
\text { - Discapacidad funcional moderada } \\
\text { - Inestabilidad unilateral de leve a } \\
\text { moderada }\end{array}$ \\
\hline $\begin{array}{l}\text { Grado 3: grave } \\
\text { Lesión completa y pérdida de la } \\
\text { integridad del ligamento en la que } \\
\text { los ligamentos están } \\
\text { completamente desgarrados y no } \\
\text { son funcionales. Lesión total } \\
\text { (ruptura) }\end{array}$ & $\begin{array}{l}\text { - Pérdida de la función y el movimiento } \\
\text { - El lesionado es incapaz de utilizar la } \\
\text { articulación afectada } \\
\text { - Dolor intenso } \\
\text { - Edema severo } \\
\text { - Equimosis severa }\end{array}$ \\
\hline
\end{tabular}


Albert J. Macías-Peralta; Stephane P. Guerrero-Zambrano; Lisbeth E. Alvear-Tóala; Raisa S. Castro-Castro; Castulo L. Moreira-Moreira; José F. Macias-Riera

Fuente: (González García, 2013)

Los expertos en la mayoría de los casos recetan como tratamiento para los esguinces: el reposo para evitar movilizar la articulación lesionada, el hielo como medio para aliviar el dolor y bajar la hinchazón, la compresión mediante vendajes sin cortar el flujo sanguíneo y la elevación con el fin de disminuir la inflamación y reducir los hematomas; este tratamiento es conocido con el acrónimo en inglés de R.I.C.E. La indicación de medicamentos es variable según el grado del esguince; el médico tratante le indicara al paciente un calmante más fuerte o menos fuerte según sea la intensidad del dolor al igual que antiinflamatorios. (Méndez Flores, 2018)

Fig.1 Tratamiento temprano de las lesiones

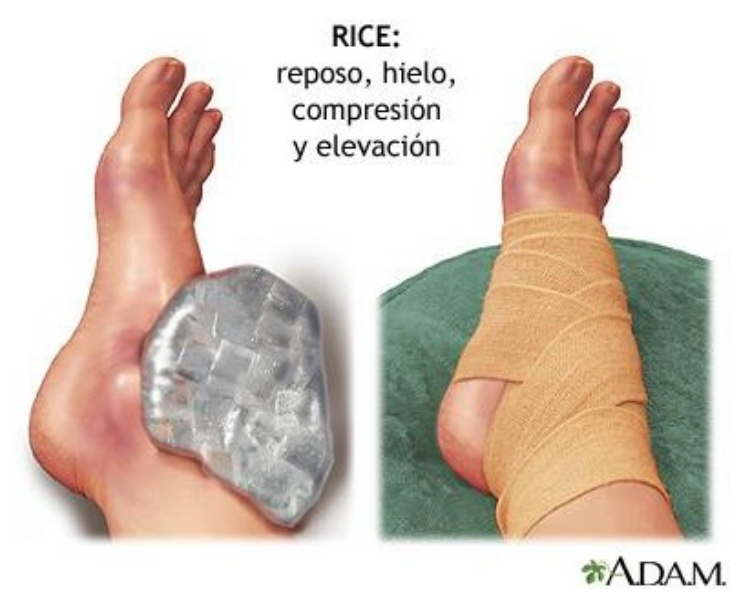

Fuente: (A.D.A.M. Inc, 2017).

Los estudios demuestran que el esguince de tobillo es una lesión que se presenta con más frecuencia en comparación a otros tipos de esguince. Según (Soria, 2015) existen personas con gran probabilidad a sufrir un esguince de tobillo:

- Las que tienen alteraciones anatómicas (deformidades) del pie.

- Las que tienen sobrepeso u obesidad. 
- Aquellas que realizan ciertas actividades laborales y deportivas riesgosas.

- Quienes padecen dolor o desequilibrio por una enfermedad del sistema nervioso central o periférico.

- Quienes tienen trombosis venosa (enfermedad de las venas).

- Las que sufren algunos trastornos de la sangre.

- Quienes ingieren de manera crónica medicamentos antiinflamatorios o anticoagulantes, entre otros.

Se entiende que el esguince debe tener ciertas y determinadas consideraciones según sea su gravedad; cada persona evoluciona de formas distintas y esto va a influenciar de forma significativa. El objetivo de este trabajo investigativo se enfoca en manejar de la mejor manera el tratamiento del esguince al momento de enfrentar este tipo de lesiones; con los conocimientos apropiados se obtendrá una mejor recuperación del paciente.

\section{Metodología.}

Para el desarrollo de este proceso investigativo, se plantea como metodología la encaminada hacia una orientación científica particular que se encuentra determinada por la necesidad de indagar en forma precisa y coherente una situación, en tal sentido (Davila, 2015) define la metodología "como aquellos pasos previos que son seleccionados por el investigador para lograr resultados favorables que le ayuden a plantear nuevas ideas". (p.66)

Lo citado por el autor, lleva a entender que el desarrollo de la acción investigativa busca simplemente coordinar acciones enmarcadas en una revisión bibliográfica con el fin de complementar ideas previas relacionadas Consideraciones frente la gravedad de problemas de esguince en el pacientea través de una revisión de literatura, para así finalmente elaborar un cuerpo de consideraciones generales que ayuden a ampliar el interés propuesto.

\section{Tipo de Investigación.}

Dentro de toda práctica investigativa, se precisan acciones de carácter metodológico mediante las cuales, se logra conocer y proyectar los eventos posibles que la determinan, así como las características que hacen del acto científico un proceso interactivo ajustado a una realidad posible de ser interpretada. En este sentido, se puede decir, que la presente investigación corresponde al tipo documental, definido por (CASTRO, 2016), "se ocupa del estudio de problemas planteados a nivel 
teórico, la información requerida para abordarlos se encuentra básicamente en materiales impresos, audiovisuales y /o electrónicos”. (p.41).

En consideración a esta definición, la orientación metodológica permitió la oportunidad de cumplir con una serie de actividades inherentes a la revisión y lectura de diversos documentos donde se encontraron ideas explicitas relacionadas con los tópicos encargados de identificar a cada característica insertada en el estudio. Por lo tanto, se realizaron continuas interpretaciones con el claro propósito de revisar aquellas apreciaciones o investigaciones propuestas por diferentes investigadores relacionadas con el tema de interés, para luego dar la respectiva argumentación a los planteamientos, en función a las necesidades encontradas en la indagación.

\section{Fuentes Documentales.}

El análisis correspondiente a las características que predomina en el tema seleccionado, llevan a incluir diferentes fuentes documentales encargadas de darle el respectivo apoyo y en ese sentido cumplir con la valoración de los hechos a fin de generar nuevos criterios que sirven de referencia a otros procesos investigativos. Para (CASTRO, 2016) las fuentes documentales incorporadas en la investigación documental o bibliográfica, "representa la suma de materiales sistemáticos que son revisados en forma rigurosa y profunda para llegar a un análisis del fenómeno”.(p.41). Por lo tanto, se procedió a cumplir con la realización de una lectura previa determinada para encontrar aquellos aspectos estrechamente vinculados con el tema, con el fin de explicar mediante un desarrollo las respectivas apreciaciones generales de importancia.

\section{Técnicas para la Recolección de la Información.}

La conducción de la investigación para ser realizada en función a las particularidades que determinan a los estudios documentales, tiene como fin el desarrollo de un conjunto de acciones encargadas de llevar a la selección de técnicas estrechamente vinculadas con las características del estudio. En tal sentido, (Bolivar, 2015), refiere, que es "una técnica particular para aportar ayuda a los procedimientos de selección de las ideas primarias y secundarias”. (p. 71).

Por ello, se procedió a la utilización del subrayado, resúmenes, fichaje, como parte básica para la revisión y selección de los documentos que presentan el contenido teórico. Es decir, que mediante la aplicación de estas técnicas se pudo llegar a recoger informaciones en cuanto a la revisión bibliográfica de los diversos elementos encargados de orientar el proceso de investigación. Tal como 
lo expresa, (Bolivar, 2015) "las técnicas documentales proporcionan las herramientas esenciales y determinantes para responder a los objetivos formulados y llegar a resultados efectivos" (p. 58). Es decir, para responder con eficiencia a las necesidades investigativas, se introdujeron como técnica de recolección el método inductivo, que hizo posible llevar a cabo una valoración de los hechos de forma particular para llegar a la explicación desde una visión general.

Asimismo, se emplearon las técnicas de análisis de información para la realización de la investigación que fue ejecutada bajo la dinámica de aplicar diversos elementos encargados de determinar el camino a recorrer por el estudio, según, (Bolívar, 2015) las técnicas de procesamiento de datos en los estudios documentales "son las encargadas de ofrecer al investigador la visión o pasos que debe cumplir durante su ejercicio, cada una de ellas debe estar en correspondencia con el nivel a emplear" (p. 123). Esto indica, que para llevar a cabo el procesamiento de los datos obtenidos una vez aplicado las técnicas seleccionadas, tales como: fichas de resumen, textual, registros descriptivos entre otros, los mismos se deben ajustar al nivel que ha sido seleccionado.

\section{Resultados.}

El tipo de lesiones como lo son los esguinces dan lugar a distintos tipos de estudios e investigaciones con el fin de mostrar que tan frecuentes son y ofrecer mejores tratamientos al paciente y de este modo obtener una recuperación más rápida.

Tener problemas en el tobillo es algo muy frecuente y se debe a que es una de las articulaciones que más peso soporta del cuerpo. El esguince de tobillo es, a su vez, la lesión más común, aunque los esguinces de muñeca o rodilla también son bastante frecuentes. En España, aproximadamente seis de cada 1.000 personas acuden cada año al médico de cabecera o al servicio de urgencias del hospital con un esguince de tobillo producido durante la práctica de algún deporte. (Medicos y Pacientes, 2009)

El problema que plantean los esguinces de tobillo es que, aparte del esguince en sí, puede haber también lesiones asociadas que pueden dificultar su tratamiento, y de las más importantes de estas son las fracturas. Las fracturas asociadas a menudo son difíciles de distinguir, ya que, se pueden confundir con el dolor que produce el esguince en sí, por lo que a menudo el diagnóstico de esguince de tobillo suele ir acompañado de una radiografía que descarte la fractura. Pero ¿qué se puede hacer si no se tiene acceso a esta prueba?

Una de las formas de disminuir la necesidad de realizar esta prueba son las reglas de Ottawa, descritas 
por primera vez en 1992 por Stiell y cols. Estas reglas permitieron reducir el número de radiografías en torno a un 30-40\%. Además, tienen una alta sensibilidad $(96,8 \%)$ lo que implica que hay menos de un $4 \%$ de fracturas que pueden ser pasadas por alto.

Las reglas de Ottawa son sencillas y fáciles de recordar y con usarlas se evitan sustos. Como mejor se aprenden y utilizan es mediante imágenes como esta:

Fig. 2 Reglas de Ottawa

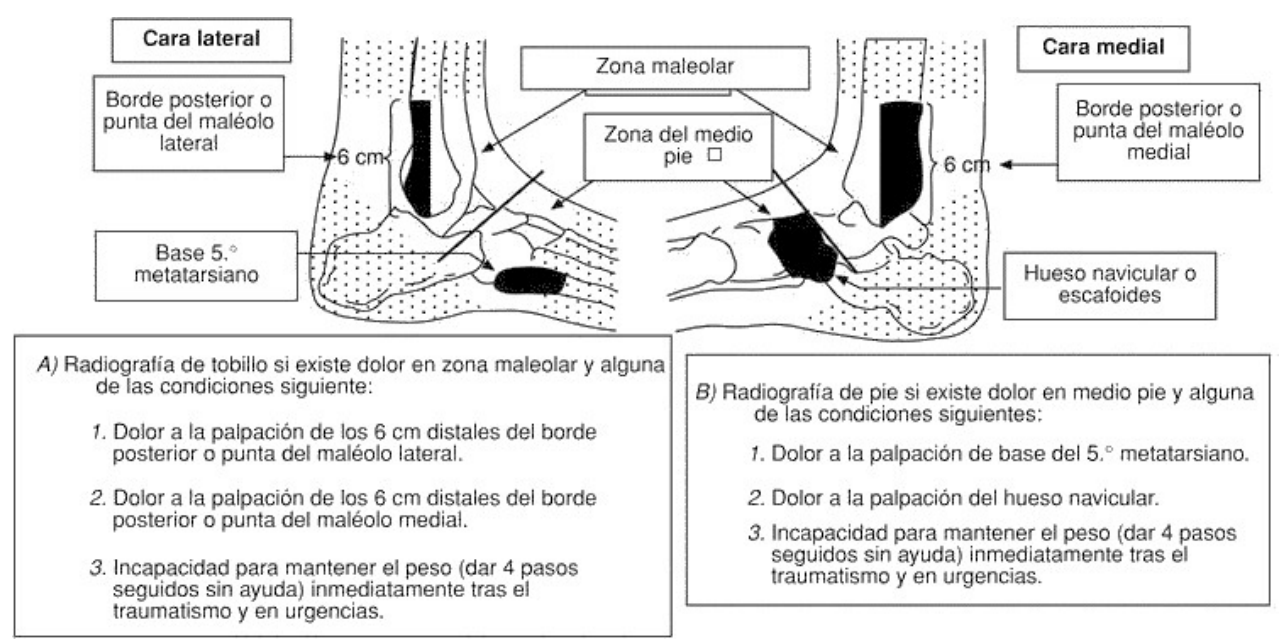

Fuente: (Rodríguez López, 2015).

La parte negativa de estas reglas es que, aunque tienen muy buena sensibilidad, su especificidad es de solo un 45,8\%. Para mejorar la especificidad de esta regla de decisión se puede usar la prueba del diapasón (tuning fork test), que consiste en producir mediante un diapasón una vibración en distintas zonas óseas para ver si el paciente tiene dolor y por tanto hay fractura. De nuevo esta prueba tiene sus limitaciones, pero reduce la necesidad de realizar radiografias. Combinando las reglas de Ottawa con la prueba del diapasón sigue siendo posible que se pase por alto alguna fractura, pero disminuyen las posibilidades de que esto ocurra. (Rodríguez López, 2015).

En un trabajo de investigación sobre la aplicación de las Reglas de Ottawa dirigido al diagnóstico de esguince de tobillo realizado por (Bustamante Vásquez, 2018) presenta como resumen que el estudio comprendió un total de 184 pacientes, que tenían un diagnóstico de esguince de tobillo independientemente de si utilizaban los criterios de Ottawa. Los datos obtenidos se tabularon y trazaron en Microsoft Excel 2013, luego se compararon con otros estudios relacionados, lo que 
demuestra que los hombres fueron los que tuvieron la mayor incidencia de esguince de tobillo, constituyendo el 54.3\%. El principal mecanismo de producción fue la caída $(61,40 \%)$.

En las siguientes imágenes se observa en detalle un esguince de tobillo en sus distintos tipos o grados siendo este uno de los más comunes:

Fig.3Anatomía normal

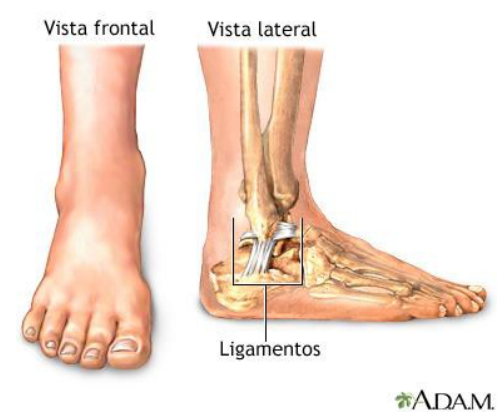

Fig.5Esguince de tobillo tipo II

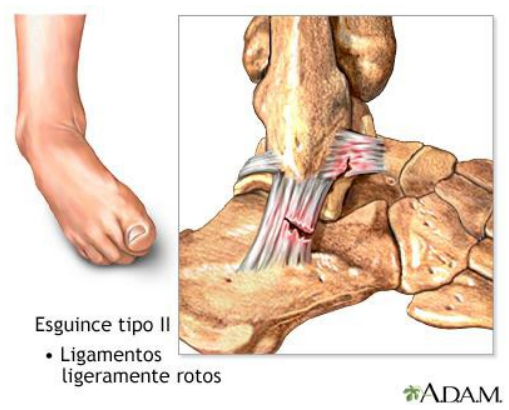

Fig.4 Esguince de tobillo tipo I

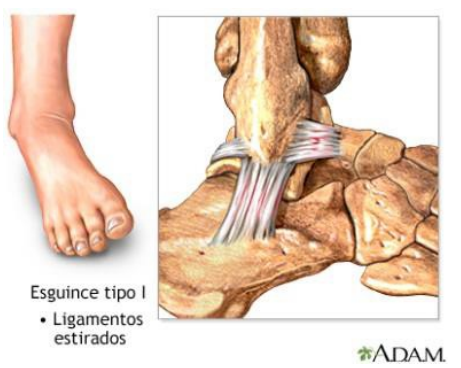

Fig.6Esguince de tobillo tipo III

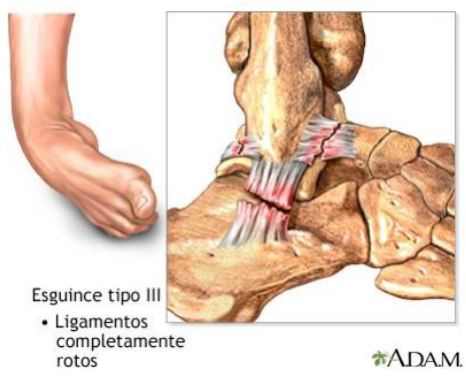

Fuente: (A.D.A.M, Inc., 2017)

Los esguinces en su mayoría presentan una lesión en los ligamentos ya sea una ruptura, estiramiento o desgarre, esto va a depender de la articulación afectada (muñeca, rodilla, pulgar) como bien se sabe que los ligamentos son las bandas elásticas que unen los huesos entre sí. A continuación, se puede hacer referencia de las causas en cada uno los casos:

Los esguinces de muñeca suelen deberse a caer sobre la mano extendida. Cualquier persona puede tener un esguince de muñeca. Pero esta lesión es más probable en personas que son muy activas o que practican deportes (UChicago Medicine, 2016).

La mayoría de esguinces de rodilla se producen como resultado de:

- Impacto directo sobre la rodilla de una fuerza exterior 
- Presión en la rodilla resultante de una fuerza de frenado brusco o cambio en la dirección

- Hiperextensión de la articulación de la rodilla

Los esguinces de rodilla son comunes en las personas que participan en deportes de ritmo rápido, tales como fútbol, rugby, baloncesto o hockey. Por ejemplo, un jugador de fútbol podría producirse un esguince de una rodilla por un cambio súbito de dirección cuando tiene un pie plantado, o también cuando con el pie plantado otro jugador cae encima de su rodilla. (terapiadefrio.com, 2018)

La mayoría de los esguinces del pulgar afectan al ligamento principal en la base del pulgar en la parte interna del dedo. Estos ligamentos se lesionan cuando el sujeto se cae sobre la mano mientras sostiene un bastón de esquí, a este tipo de lesión se le denomina pulgar del esquiador. El ligamento también puede lesionarse cuando el pulgar queda atrapado y se desplaza hacia atrás sobre una superficie dura al caer o al ser golpeado durante una captura de una bola de béisbol. El estiramiento excesivo repetido de la articulación también puede desgarrar el ligamento, como ocurría antiguamente cuando los guardabosques ingleses rompían el cuello de los conejos con su mano, por este motivo a esta lesión también se le conoce con el nombre de pulgar de guardabosques. (Campagne, 2015)

En lo que se refiere a la sintomatología es básicamente la misma en los tres casos: dolor: este puede ser variable según sea la gravedad de la lesión; hinchazón o inflamación, hematomas o moretones, imposibilidad para desenvolverse en las actividades cotidianas; entre otros.

El tratamiento de un esguince de muñeca puede incluir lo siguiente:

- Medicamentos recetados o de venta libre. Ayudan a reducir el dolor y la inflamación.

- Una férula, un yeso u otro elemento ortopédico. Se usa para sostener la muñeca y evitar que se mueva. Puede que necesite usar este elemento por algunas semanas o más hasta tanto la muñeca se recupere.

- Fisioterapia y ejercicios. Ayudan a mejorar la fuerza, la flexibilidad y la amplitud de movimiento de su muñeca y su mano.

- Cirugía. Puede que necesite cirugía si su esguince es grave. Por ejemplo, si el ligamento está desgarrado o si también se han lesionado otros tejidos y el hueso de la zona. Después de la cirugía, con frecuencia necesitará usar una férula o un yeso por un mes o más hasta tanto sane su muñeca. (UChicago Medicine, 2016) 
Para determinar el tratamiento de esguince de pulgar se aplicarán radiografías que pueden mostrar si los huesos del pulgar y de la mano están rotos. El especialista en la mano le examinará el pulgar para determinar si el ligamento está desgarrado. Algunos desgarros se pueden tratar usando una tablilla o un yeso. Otros desgarros tienen que arreglarse quirúrgicamente.

Si la lesión es crónica, el ligamento podría no sanarse ni siquiera con tratamiento. En esos casos, a veces se crea un nuevo ligamento usando un tendón. Si en la articulación hay artritis, la mejor opción podría ser fusionarla para que no se mueva en absoluto. (ASSH, 2015).

El tratamiento que se lleva a cabo en el caso de un esguince de rodilla en el siguiente:

- A veces, drenaje del líquido articular.

- Protección, reposo, hielo, compresión y elevación.

- Una férula o un inmovilizador (ortesis) de rodilla y muletas.

Si se ha producido un derrame significativo de líquido en la rodilla, en algunas ocasiones el médico puede drenarlo para aliviar el dolor. La mayoría de las lesiones leves o moderadas se pueden tratar inicialmente con protección, reposo, hielo, compresión, elevación (protection, rest, ice, compression, and elevation, PRICE) e inmovilización de la rodilla con una férula o con un dispositivo que proporcione apoyo a la rodilla y evite que se doble (inmovilizador de rodilla).

Si el esguince es grave, algunos sujetos necesitan usar un inmovilizador de rodilla durante 4 a 6 semanas o más. Algunas lesiones graves de ligamentos o meniscos requieren una reparación quirúrgica. Este procedimiento generalmente se realiza a través de una pequeña incisión utilizando un pequeño tubo flexible (lo que se denomina cirugía artroscópica). En personas con lesiones leves o moderadas se pueden recomendar ejercicios para fortalecer la rodilla. Si las lesiones son graves, los ejercicios de fortalecimiento se posponen hasta después de la cirugía. (Campagne, 2015)

Al momento de realizar alguna actividad física se deben tomar todas las previsiones posibles para evitar una lesión de este tipo; en especial si se practica algún deporte que tenga posibilidad de generar esguince. En muchos casos esto puede ser fortuito si se trata de una caída o accidente laboral. Pero si se puede evitar teniendo en cuenta una serie de consideraciones:

$\checkmark \quad$ Evitar en lo posible las caídas.

$\checkmark$ Hacer ejercicio luego de un adecuado calentamiento. 
$\checkmark$ Alimentarse de forma sana.

$\checkmark$ Utilizar zapatos adecuados para la actividad a realizar.

$\checkmark$ Correr en superficies planas.

$\checkmark$ Mantener un peso propio para la edad y las condiciones de la persona

$\checkmark$ Entre otras.

\section{Conclusión.}

Cuando se va ejecutar cualquier labor, profesión o tarea que pueda ocasionar una lesión en donde se vea comprometido el desenvolvimiento de la vida diaria, ésta se debe tomar con mucha seriedad; y un esguince es una de ellas, este tipo de lesión puede generar consecuencias que no permitirán el correcto funcionamiento del miembro afectado, de no ser consiente con la gravedad de la lesión ysi no se tiene el debido reposo. Es muy importante tratar lo antes posible la articulación que presenta el esguince y acudir al médico.

Como se ha visto existen diversos tipos de esguinces siendo el de tobillo uno de los más frecuentes; y con mayor presencia en el ámbito deportivo; donde se presenta con una incidencia de 1 de cada 1000 personas/día. Aunque también se pueden presentar esguinces en rodillas, codos, dedos y muñecas.

De acuerdo con los estudios mencionados en los resultados los esguinces tienden a presentar la misma sintomatología: dolor fuerte, hinchazón, hematomas, imposibilidad de utilizar el miembro afectado, y en ocasiones deformación.

Las consideraciones que se deben tener ante un problema de esguince en un paciente son básicas y se pueden resumir en las siglas R.I.C.E (REPOSO, HIELO (ICE), COMPRESION y ELEVACION); estas aplican para la mayoría de los casos según sea la gravedad de la lesión. También es importante que se tengan en cuenta que mantener una buena alimentación y una vida activa puede evitar que ocurra una lesión, utilizar un buen calzado y el equipo adecuado son indispensables.

Por otra parte, se puede mencionar que las estadísticas demuestran que el esguince de tobillo es el más común; este tipo de esguince tiende a afectar en su mayoría a deportistas; con suerte estos cumplen con los tratamientos y terapias adecuadas y logran superar este tipo de lesiones. Los expertos en este tema siempre aconsejan cumplir con las indicaciones del médico tratante. De esta manera al igual que un deportista una persona puede superar un esguince. 
Además del esguince de tobillo son frecuente las lesiones de rodilla, muñeca y pulgar quizás no con la misma incidencia. En los tres casos se presentan casi los mismos síntomas y se pueden manejar con las mismas consideraciones al momento de aplicar el tratamiento de primera mano (RICE). No se debe ejercer ningún esfuerzo innecesario en la extremidad afectada y se debe guardar el reposo indicado por el médico.

\section{Bibliografía.}

A.D.A.M. Inc. (14 de mayo de 2017). Medline Plus. Recuperado el 30 de mayo de 2019, de https://medlineplus.gov/spanish/ency/esp_imagepages/19396.

ASSH. (2015). American Society for Surgery of the Hand. Recuperado el 30 de mayo de 2019, de https://www.assh.org/handcare/espanol/esguinces-del-dedo-pulgar-thumbsprain\#prettyPhoto: https://www.assh.org/handcare/

Bolívar, J. (2015). Investigación Documental. México. Pax.

Bustamante Vásquez, C. A. (2018). Costo efectividad en el diagnóstico y manejo de esguince de tobillo, basados en los criterios de Ottawa durante los años 2014 y 2015 en el Hospital Isidro Ayora. Loja, Ecuador.

Campagne, D. (enero de 2015). Manual traumatiso y envenamiento, fracturas luxaciones y esguinces. Lima . Recuperado el 30 de mayo de 2019, de https:/www.msdmanuals.com/esve/hogar/traumatismos-y-envenenamientos/fracturas,-luxaciones-y-esguinces/esguinces-delpulgar: https://www.msdmanuals.com/es-ve/\#

Castro, J. (2016). Técnicas Documentales. México. Limusa.

Davila, A. (2015). Diccionario de Términos Científicos. . Caracas: Editorial Oasis.

González García, A. (2013). Manual de Primeros Auxilios para enfermería. En, A. Díaz, \& D. Santos, Manual de Primeros Auxilios para enfermería (pág. 128). DAE.

Martínez, S. (2019). https://amplab-fisio.com/. Recuperado el 29 de mayo de 2019, de https://amplabfisio.com/esguince-de-tobillo-actualizacion-y-consideraciones-biomecanicas/: https://amplab-fisio.com/ 
Albert J. Macías-Peralta; Stephane P. Guerrero-Zambrano; Lisbeth E. Alvear-Tóala; Raisa S. Castro-Castro; Castulo L. Moreira-Moreira; José F. Macias-Riera

Medicos y Pacientes. (27 de junio de 2009). Recuperado el 29 de mayo de 2019, de http://www.medicosypacientes.com/articulo/consejos-para-tratar-un-esguince-de-tobillo: http://www.medicosypacientes.com

Méndez Flores, A. (2018). Ciencias Medicas. Bogota. Recuperado el 29 de mayo de 2019, de https://blog.ciencias-medicas.com/archives/1795: https://blog.ciencias-medicas.com/

Rodríguez López, Ó. (21 de mayo de 2015). Esguince de tobillo y reglas de ottawa. Revista Medica Panamericana.com/, 56-63. Recuperado el 30 de mayo de 2019, de http://blog.medicapanamericana.com/esguince-de-tobillo-y-reglas-de-ottawa/: http://blog.medicapanamericana.com/

Soria, E. S. (22 de octrubre de 2015). Galenored Internacional. Recuperado el 30 de mayo de 2019, de https://galenored.com/los-esguinces/: https://galenored.com/

terapiadefrio.com. (04 de junio de 2018). Recuperado el 30 de mayo de 2019, de https://www.terapiadefrio.com/blogs/terapia-de-frio-calor-tratamiento-natural-delesiones/sintomas-causas-y-factores-de-riesgo-en-esguinces-de-rodilla: https://www.terapiadefrio.com/

UChicago Medicine. (03 de octubre de 2016). Recuperado el 30 de mayo de 2019, de http:/healthlibrary.uchospitals.edu/spanish/video-library/Content/healthsheetsv1/understanding-a-wrist-sprain-1/: https://www.uchicagomedicine.org/ 